\title{
Anticancer effects of oridonin on colon cancer are mediated via BMP7/p38 MAPK/p53 signaling
}

\author{
RONG-XING LIU ${ }^{1,2^{*}}$, YAN MA ${ }^{1,2^{*}}$, XUE-LIAN HU ${ }^{1,3^{*}}$, WEN-YAN REN $^{1,2}$, YUN-PENG LIAO ${ }^{1,2}$, \\ $\mathrm{HAN} \mathrm{WANG}^{1,2}$, JIA-HUI ZHU ${ }^{1,2}, \mathrm{KE} \mathrm{WU}^{1,2}$, BAI-CHENG HE ${ }^{1,2}$ and WEN-JUAN SUN ${ }^{1,2}$ \\ ${ }^{1}$ Department of Pharmacology, School of Pharmacy, and ${ }^{2}$ Key Laboratory for Biochemistry and \\ Molecular Pharmacology of Chongqing, Chongqing Medical University, Chongqing 400016; \\ ${ }^{3}$ Department of Pharmacy, Xinqiao Hospital, Chongqing 400037, P.R. China
}

Received April 6, 2018; Accepted August 7, 2018

DOI: $10.3892 /$ ijo.2018.4527

\begin{abstract}
Colon cancer is a prevalent malignancy affecting the gastrointestinal tract. Oridonin (ORI) is a promising chemotherapeutic drug used in the treatment of colon cancer. In this study, we examined the anticancer activity of ORI against colon cancer and elucidated the underlying molecular mechanisms. Cell counting kit-8, flow cytometric and western blot analyses were conducted to analyze the growth inhibitory effects of ORI on SW620 cells; we employed BMP7 and p53 recombinant adenovirus to detect the influence of ORI on the p38 MAPK signal pathway; PT-qPCR, cell immunofluorescence staining and western blot analysis were used to detect the expression of BMP7, p38 and p-p38, p53 and p-p53. A xenograft tumor model and histological evaluation were introduced to detect the effects of ORI and BMP7 in SW620 cells in vivo. ORI inhibited the proliferation of SW620 cells and induced apoptosis. ORI also increased the total and phosphorylated levels of p53. The overexpression of p53 was found to enhance the anti-proliferative effects of ORI on the SW620 cells, while the inhibition of p53 partially reversed these effects. ORI increased the expression of bone morphogenetic protein 7 (BMP7) in the SW620 cells. The overexpression of BMP7 also enhanced the antiproliferative effects of ORI on the SW620 cells and reduced the growth rate of tumors in mice. BMP7-induced immunosuppression markedly decreased the anti-proliferative effects of ORI. ORI was not found to exert any substantial effect on the phosphorylation levels of Smad1/5/8, although
\end{abstract}

Correspondence to: Professor Wen-Juan Sun or Professor Bai-Cheng He, Department of Pharmacology, School of Pharmacy, Chongqing Medical University, 1 Yixueyuan Road, Yuzhong, Chongqing 400016, P.R. China

E-mail: 1115494605@qq.com

E-mail: hebaicheng99@yahoo.com; 894704897@qq.com

*Contributed equally

Key words: oridonin, colon cancer, bone morphogenetic protein 7 , p38 mitogen-activated protein kinase, p53 it increased the level of p-p38 significantly. The inhibition of p38 significantly attenuated the ORI-induced increase in the levels of p-p53. The overexpression of BMP7 enhanced the promoting effects of ORI on the p-p53 and p-p38 levels, while BMP7-induced immunosuppression reduced the effects of ORI on p-p38 and p-p53. On the whole, the findings of this study suggest that ORI may be a promising agent for use in the treatment of colon cancer, and the anticancer effects of ORI may be partially mediated through the BMP7/p38 MAPK/p53 signaling pathway.

\section{Introduction}

Colon cancer, an aggressive malignancy causes substantial morbidity and mortality $(1,2)$. The current treatment for patients with colon cancer relies on chemotherapy, despite its severe side-effects. Antineoplastic drugs from natural resources (e.g., camptothecin and vincristine) are extensively utilized in the treatment of colon cancer $(3,4)$. Oridonin (ORI), a vital diterpenoid from the Chinese herbal medicine, Rabdosia rubescens (5) can effectively be utilized in the treatment of breast cancer, colon cancer, osteosarcoma and prostate cancer (5-8). However, the mechanisms underlying the effects of ORI remain unclear.

Bone morphogenetic proteins (BMPs), a subgroup of the transforming growth factor (TGF)- $\beta$ superfamily are associated with multiple physiological functions, including the regulation of cell differentiation, proliferation and apoptosis (9-11). BMP2, BMP4 and BMP7 can inhibit the proliferation of colon cancer cells $(9,12)$. BMP2 and BMP4 can even trigger the differentiation of colon cancer stem cells (13). BMP7 can mediate the anticancer activity of ORI by activating p38 mitogen-activated protein kinase (MAPK) and thereby enhancing the function of phosphatase and tensin homolog (PTEN) $(7,14)$. However, the detailed underlying mechanisms remain unclear.

p53, a well-known tumor suppressor and a critical mediator of the cellular stress response, is considered as a valid therapeutic target (15-18). The functional loss or mutation of p53 is regarded as the primary cause of cancer. MAPK is another crucial cell-growth regulator in the pathogenesis of cancer (19). Aberrant p38 MAPK signals have been noted in 
solid tumors, such as breast cancer and colon cancer (20). p38 MAPK regulates the p53 signal (21). Thus, it is possible that BMP7 also regulates the activity of p53 in colon cancer cells.

In the present study, in vitro, and in vivo assays were performed to investigate the potential of p53 to mediate the antiproliferative activity of ORI against colon cancer cells and to elucidate the possible mechanistic association between BMP7 and $\mathrm{p} 53$.

\section{Materials and methods}

Reagents and cell culture. ORI (procured from Hao-Xuan Bio-Tech Co., Ltd., Xi'an, China) was dissolved in DMSO at the concentration of $10 \mathrm{mmol} / 1$ and stored at $-20^{\circ} \mathrm{C}$. For the in vivo experiments, an ORI suspension was prepared with a $0.4 \%$ carboxymethylcellulose sodium (CMC-Na) solution. All primary antibodies used in this study were purchased from Santa Cruz Biotechnology, Inc. (Santa Cruz, CA, USA). The inhibitors of p53 [pifithrin- $\beta$ hydrobromide (PFT) cat. no. S2929] and p38 (SB203580; cat. no. S1076) were purchased from Shanghai Selleck Chemicals Co., Ltd. (Shanghai, China). All the cell lines used (HCT116, SW620, SW480 LoVo and FHC) were obtained from the American Type Culture Collection (ATCC, Manassas, VA, USA). The cells were cultured in Dulbecco's modified Eagle's medium with $10 \%$ fetal bovine serum, $100 \mathrm{U} / \mathrm{ml}$ of penicillin and $100 \mu \mathrm{g} / \mathrm{ml}$ of streptomycin solution at $37^{\circ} \mathrm{C}$ under a $5 \% \mathrm{CO}_{2}$ environment.

Construction of BMP7 and p53 recombinant adenoviruses. The recombinant adenoviruses for BMP7 and p53 were constructed using the AdEasy system (22), tagged with green fluorescence protein (GFP) and designated as AdBMP7 or Adp53. The GFP-expressing recombinant adenovirus was used only as a vector control (AdGFP). Samples were collected at 24 or $48 \mathrm{~h}$ following transfection.

Cell viability assay. Cell viability and proliferation were determined using a cell counting kit-8 (CCK-8). Briefly, the SW620 cells were seeded onto 96 -well plates at $3 \times 10^{3}$ cells/well and treated with various concentrations of ORI solutions $(0,5$, $10,15,20$ and $25 \mu \mathrm{M})$, corresponding reagents or DMSO. After 24,48 or $72 \mathrm{~h}, 10 \mu \mathrm{l}$ of CCK-8 were added to each well followed by incubation at $37^{\circ} \mathrm{C}$ for $4 \mathrm{~h}$. The optical absorbance was determined at $450 \mathrm{~nm}$ using a microplate reader (ELx800; BioTek Instruments, Inc., Winooski, VT, USA). Each assay was conducted in triplicate.

Flow cytometric analysis of the cell cycle and apoptosis. The SW620 cells were seeded into 6-well plates and treated for $48 \mathrm{~h}$ as per protocol $(0,10,15$ and $20 \mu \mathrm{M})$. For analyzing cellular apoptosis, the cells were collected and washed with PBS $\left(4^{\circ} \mathrm{C}\right)$ and incubated with Annexin V EGFP and propidium iodide (PI; Keygenbio, Nanjing, China). The cells were then sorted following fluorescence activation. For cell cycle analysis, the cells were collected and washed with PBS $\left(4^{\circ} \mathrm{C}\right)$, fixed with chilled $\left(4^{\circ} \mathrm{C}\right) 70 \%$ ethanol, washed with 50 and $30 \%$ ethanol, and PBS. The cells were then stained with $1 \mathrm{ml}$ of PI $(20 \mathrm{mg} / \mathrm{ml})$ containing RNase $(1 \mathrm{mg} / \mathrm{ml})$ for $30 \mathrm{~min}$ and analyzed by flow cytometry (BD Influx; BD Biosciences, Franklin Lakes, NJ, USA). Each assay was carried out in triplicate.
RNA extraction, reverse transcription-quantitative $P C R$ (RT-qPCR) assay. Sub-confluent SW620 cells were plated in T25 flasks and treated with various concentrations of ORI $(0,5,10$, 15,20 and $25 \mu \mathrm{M}$ ) or corresponding reagents. Total RNA was extracted from the cells using TRIzol reagent (Invitrogen/Thermo Fisher Scientific, Waltham, MA, USA) and subjected to reverse transcription to generate cDNA using a PrimeScript RT Reagent kit (Takara, Kosatsu, Japan). The cDNA was then used as a template for the qPCR assay with 2X SYBR-Green qPCR Master Mix (Bimake, Houston, TX, USA). The PCR thermocycling conditions were as follows: $95^{\circ} \mathrm{C}$ for $1 \mathrm{~min}$ for pre-denaturation, then 30 cycles of $92^{\circ} \mathrm{C}$ for $2 \mathrm{~min}$ for denaturation, $55^{\circ} \mathrm{C}$ for $45 \mathrm{sec}$ for annealing and $72^{\circ} \mathrm{C}$ for $45 \mathrm{sec}$ for elongation, finally $72^{\circ} \mathrm{C}$ for $5 \mathrm{~min}$ for re-elongation. The primer sequences used in this study were as follows: (5'-3'): GAPDH forward, CAACGAATTTGG CTACAGCA and reverse, AGGGGAGATTCAGTGTGGTG; p53 forward, GTCGGTGGGTTGGTAGTTTCTA and reverse, AAAAAGAAATTGACCCTGAGCA; BMP7 forward, GGC AGGACTGGATCATCG and reverse, AAGTGGACCAGC GTCTGC. RT-qPCR results were analyzed by $2^{-\Delta \Delta C q}$ method described by Livak and Schmittgen (23). Each assay was carried out in triplicate.

Western blot analysis. Sub-confluent SW620 cells were seeded in 6-well plates and then treated with various concentrations of ORI $(10,15$ and $20 \mu \mathrm{M})$ or corresponding regents. At the scheduled time point ( 24 or $48 \mathrm{~h}$ ), cells were lysed with $300 \mu \mathrm{l}$ lysis buffer (10\% SD; Glycerinum; Tris-Hcl, 1 M, pH 6.8; protease inhibitor cocktail, EDTA-Free, 100X in DMSO; phosphatase inhibitor cocktail, 2 tubes, $100 \mathrm{X} ; \mathrm{H}_{2} \mathrm{O}$ ) in each well, and the lysate was boiled for $10 \mathrm{~min}$. The protein concentration was determined using the BCA Protein Assay kit, and a total of $40 \mu \mathrm{g}$ protein was loaded per lane and subjected to electrophoresis by $10 \%$ SDS-PAGE separation and transferred onto polyvinylidene fluoride membranes. The membranes were then blotted with corresponding primary antibodies against GAPDH (sc-32233-R, 1:3,000), Bad (sc-8044-M, 1:3,000), Bcl-2 (sc-7382-M, 1:3,000), p53 (sc-55476-M, 1:3,000), phospho-p53 (sc-17105-G, 1:3,000), BMP7 (sc-9305-G, 1:3,000), Smad1/5/8 (sc-6031-R, 1:3,000), phospho-Smad1/5/8 (sc-12353-G, 1:3,000), p38 (sc-535-R, 1:3,000) and phosphop38 (sc-7973-M, 1:3,000) for $12 \mathrm{~h}$ at $4^{\circ} \mathrm{C}$. The membranes were then immunoblotted with HRP-conjugated secondary antibodies (goat anti-rabbit $\operatorname{lgG}, \mathrm{SA00001-2,} \mathrm{1:3,000;} \mathrm{goat}$ anti-mouse $\operatorname{lgG}$, SA00001-1, 1:3,000; rabbit anti-goat IgG, SA00004-4, 1:3,000) successively, all antibodies used in this study were purchased from Santa Cruz Biotechnology, Inc.. The target proteins were developed with SuperSignal West Femto Substrate (\#34095; Thermo Scientifc, Rockford, IL, USA). Each assay was done in triplicate.

Cell immunofluorescence staining assay. The cells were seeded in 48-well plates and treated as per protocol $(0,10$ and $20 \mu \mathrm{M})$. At the scheduled time point $(48 \mathrm{~h})$, the cells were fixed with methanol $\left(4^{\circ} \mathrm{C}\right)$ for $15 \mathrm{~min}$, washed with PBS $\left(4^{\circ} \mathrm{C}\right)$, permeabilized with $0.5 \%$ Triton $\mathrm{X}-100$, and then blocked with 5\% BSA at room temperature for $1 \mathrm{~h}$ and incubated with the primary antibody for the proteintarget (p53, sc-55476-M, 1:100; BMP7, sc-9305-G; 1:100; Santa Cruz Biotechnology, Inc.). The corresponding IgGs 
(negative control) were incubated with FITC-conjugated with corresponding secondary antibodies (goat anti-mouse $\lg \mathrm{G}$, SA00001-1, 1:100; rabbit anti-goat IgG, SA00004-4, 1:100; Santa Cruz Biotechnology, Inc.) for $30 \mathrm{~min}$. Finally, the cells were stained with DAPI $(1 \mu \mathrm{g} / \mathrm{ml}$, Solarbio, Beijing, China). Images were recorded under an inverted microscope (Nikon ECLIPSE Ti-S; Nikon Corporation, Tokyo, Japan). Each assay was carried out in triplicate.

Xenograft tumor model for human colon cancer and histological evaluation. All animal experiments followed the guidelines of the Institutional Animal Care and Use Committee of Chongqing Medical University (Chongqing, China). Athymic nude mice (female, weighing 14-15 g, 4-6 weeks old, 5/group, 4 groups in total) were ordered and kept at the animal center of Chongqing Medical University $\left(26-28^{\circ} \mathrm{C}, 40-60 \%\right.$ relative humidity), and fed by the staff. The SW620 cells were cultured and treated with ORI and/or AdBMP7. The cells were then harvested, resuspended in PBS $\left(4^{\circ} \mathrm{C}\right)$ and injected subcutaneously ( $1 \times 10^{6} /$ injection) into the right flanks of athymic nude mice. The mice were administered intragastrically with an ORI suspension (50 or $100 \mathrm{mg} / \mathrm{kg}$ ) or the same volume of CMC-Na once daily for 3 weeks. After 3 weeks, all nude mice were sacrificed to retrieve all the tumor samples. The weights and diameters of the tumor samples were measured with digital calipers, and the volume of a tumor was calculated using the following formula: Tumor volume $\left(\mathrm{cm}^{3}\right)=$ longer diameter $x$ shorter diameter ${ }^{2} / 2$. The samples were then fixed in $10 \%$ formalin and embedded in paraffin. Serial sections of the embedded samples were stained with hematoxylin and eosin (H\&E) or immunohistochemical stains (All stains were made by School of Basic Medicine of Chongqing Medical University, Chongqing, China). Each experiment was carried out 3 times.

Statistical analysis. All data are presented as the means \pm standard deviation (SD). The differences between groups were estimated by one-way analysis of variance followed by a Dunn-Bonferroni test for multiple comparisons and a value of $\mathrm{P}<0.05$ was considered to indicate a statistically significant difference.

\section{Results}

Effects of ORI on the growth of colon cancer cells. In this study, we tried to elucidate the possible molecular mechanisms behind the anticancer effects of ORI against colon cancer. The anti-proliferative activity of ORI against the SW620 cell line was time- and concentration-dependent and significantly higher than the other cell lines (Fig. 1A), which would justify the selection of the SW620 cells for use in the following experiments. The results of the flow cytometric assay revealed that ORI arrested the cells at predominately the G2 phase of the cell cycle (Fig. 1B). These results suggest that ORI inhibits the proliferation of colon cancer cells.

Effects of ORI on the apoptosis of SW620 cells. The pro-apoptotic effects of ORI against SW620 cells were also determined. The results of flow cytometric analysis revealed the concentration-dependent pro-apoptotic potential of ORI against the
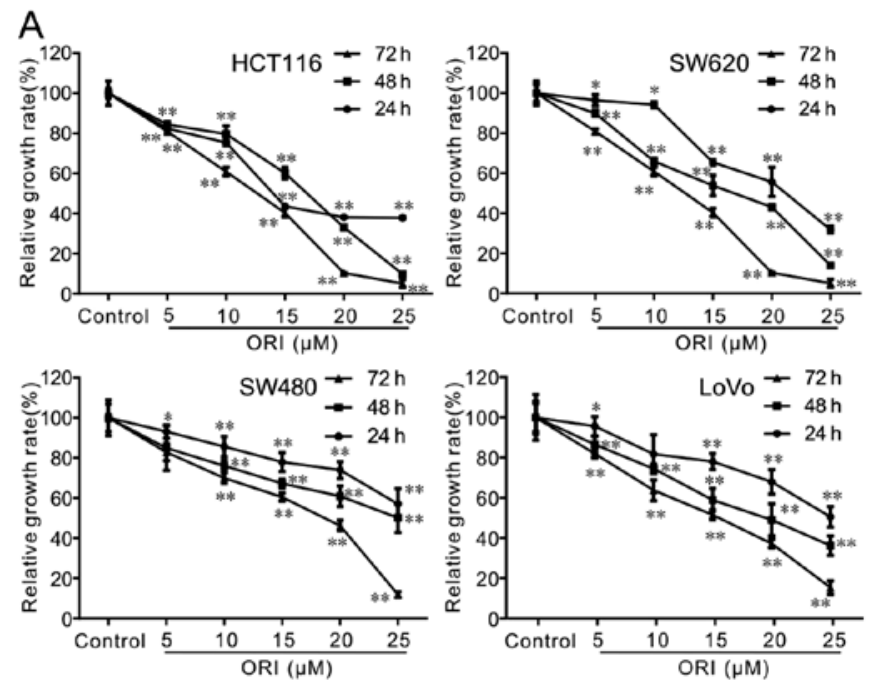

B
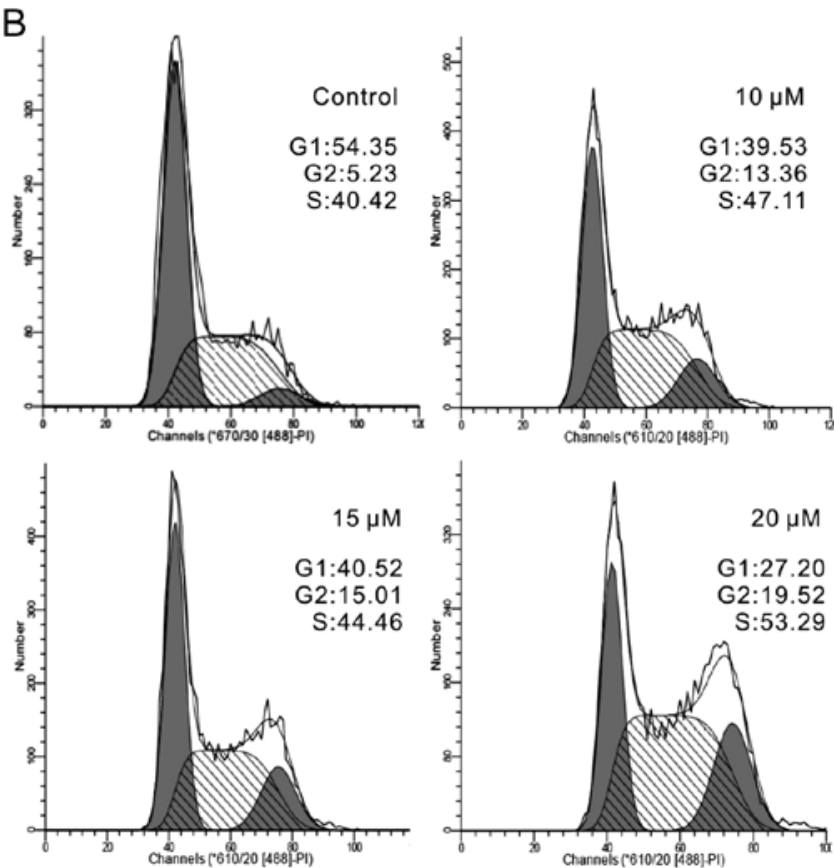

Figure 1. Effects of oridonin (ORI) on the proliferation of colon cancer cells (A) The results of CCK-8 assay revealed the effect of ORI on the proliferation of colon cancer cells, including HCT116 cells, SW620, SW480 and LoVo cells ( ${ }^{*} \mathrm{P}<0.05$ vs. control; ${ }^{* *} \mathrm{P}<0.01$ vs. control). (B) Results of flow cytometric analysis showing the effect of ORI on cell cycle arrest in SW620 cells.

colon cancer cells (Fig. 2A). ORI also increased the expression levels of Bad, and decreased those of Bcl-2 (Fig. 2B). These data suggest that ORI may be a potent inducer of the apoptosis of colon cancer cells.

Effects of ORI on the level of p53 in SW620 cells. The role of p53 in mediating the anticancer effects of ORI was also investigated in colon cancer cells. The results of the RT-qPCR assay revealed that ORI significantly upregulated the expression of p53 (Fig. 3A). The results of cell immunofluorescence staining and western blot assay analysis also revealed that ORI increased the level of p53 and p-p53 in a time- and concentration-dependent manner (Fig. 3B and C). Thus, these results suggest that ORI exerts anticancer effects on colon cancer cells through the activation of the p53 signal. 

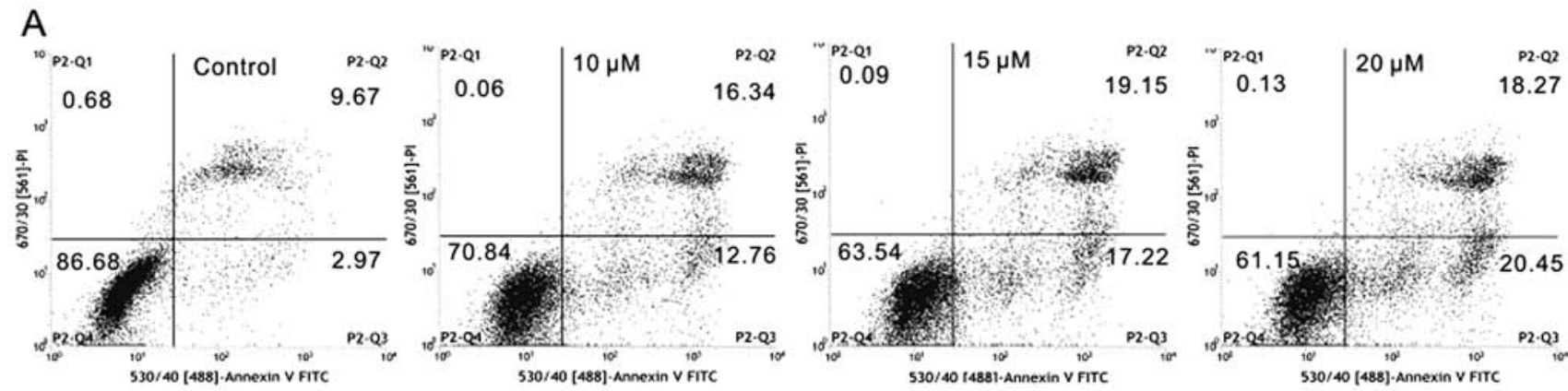

B
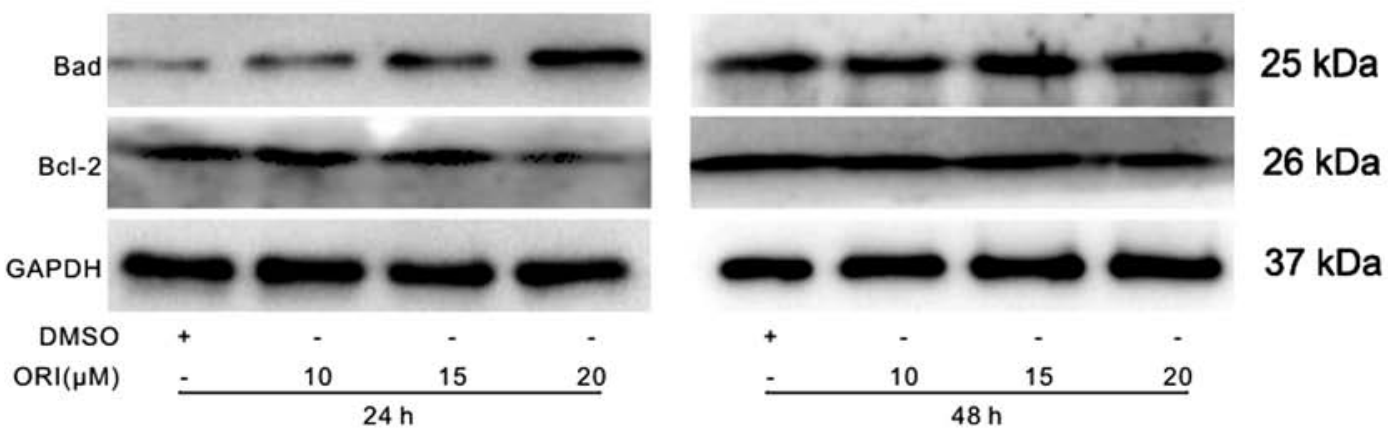

Figure 2. Effects of oridonin (ORI) on the apoptosis of SW620 cells. (A) Results of flow cytometric analysis showing the effect of ORI on the apoptosis of SW620 cells. (B) Results of western blot analysis showing the effect of ORI on the protein levels of Bad and Bcl-2 in SW620 cells.

A

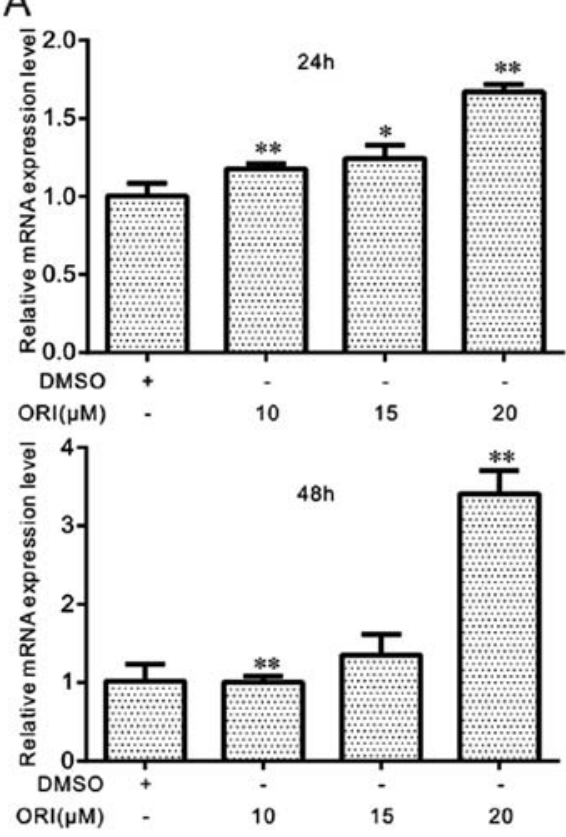

C
B

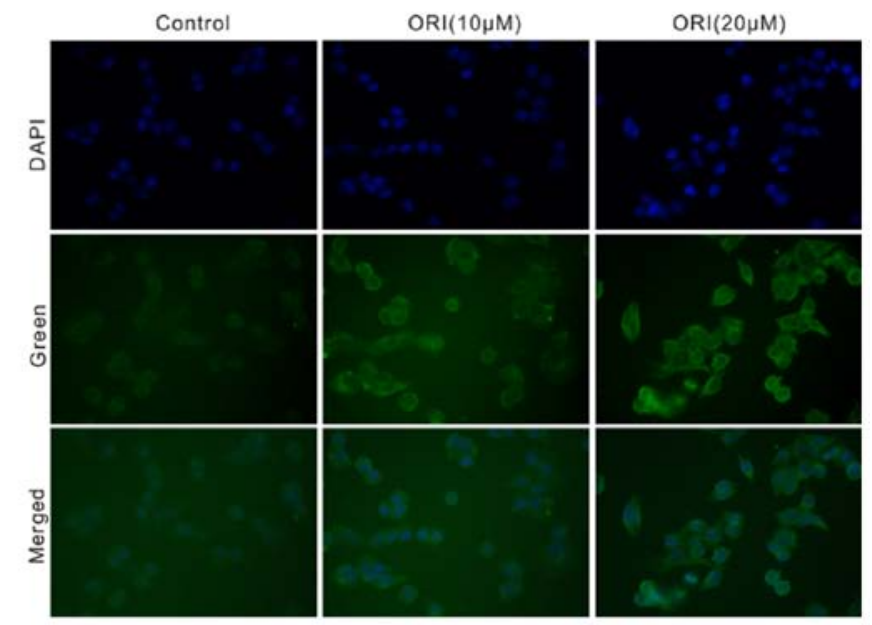

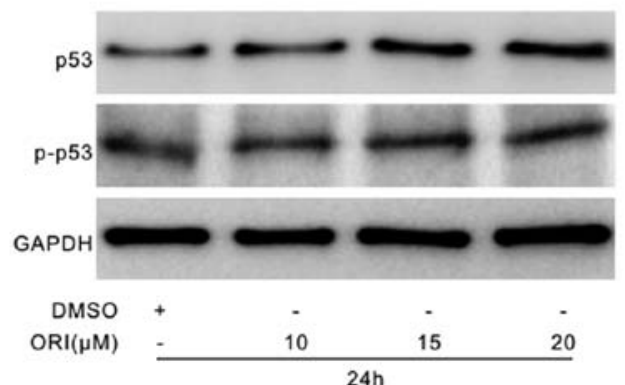

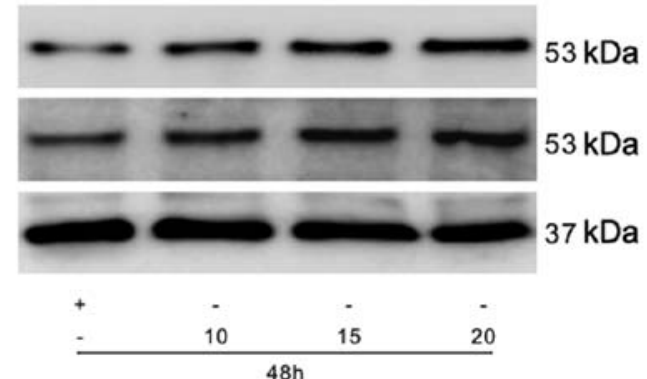

Figure 3. Effects of oridonin (ORI) on the p53 signal in SW620 cells. (A) Results of RT-qPCR assay showing the effect of ORI on the mRNA expression of p53 in SW620 cells ( ${ }^{*} \mathrm{P}<0.05$ vs. control; ${ }^{* *} \mathrm{P}<0.01$ vs. control). (B) Results of immunofluorescence staining showing the effect of ORI on p53 expression in SW620 cells. (C) Results of western blot assay analysis showing the effect of ORI on the protein level of p53 and p-p53 in SW620 cells. 

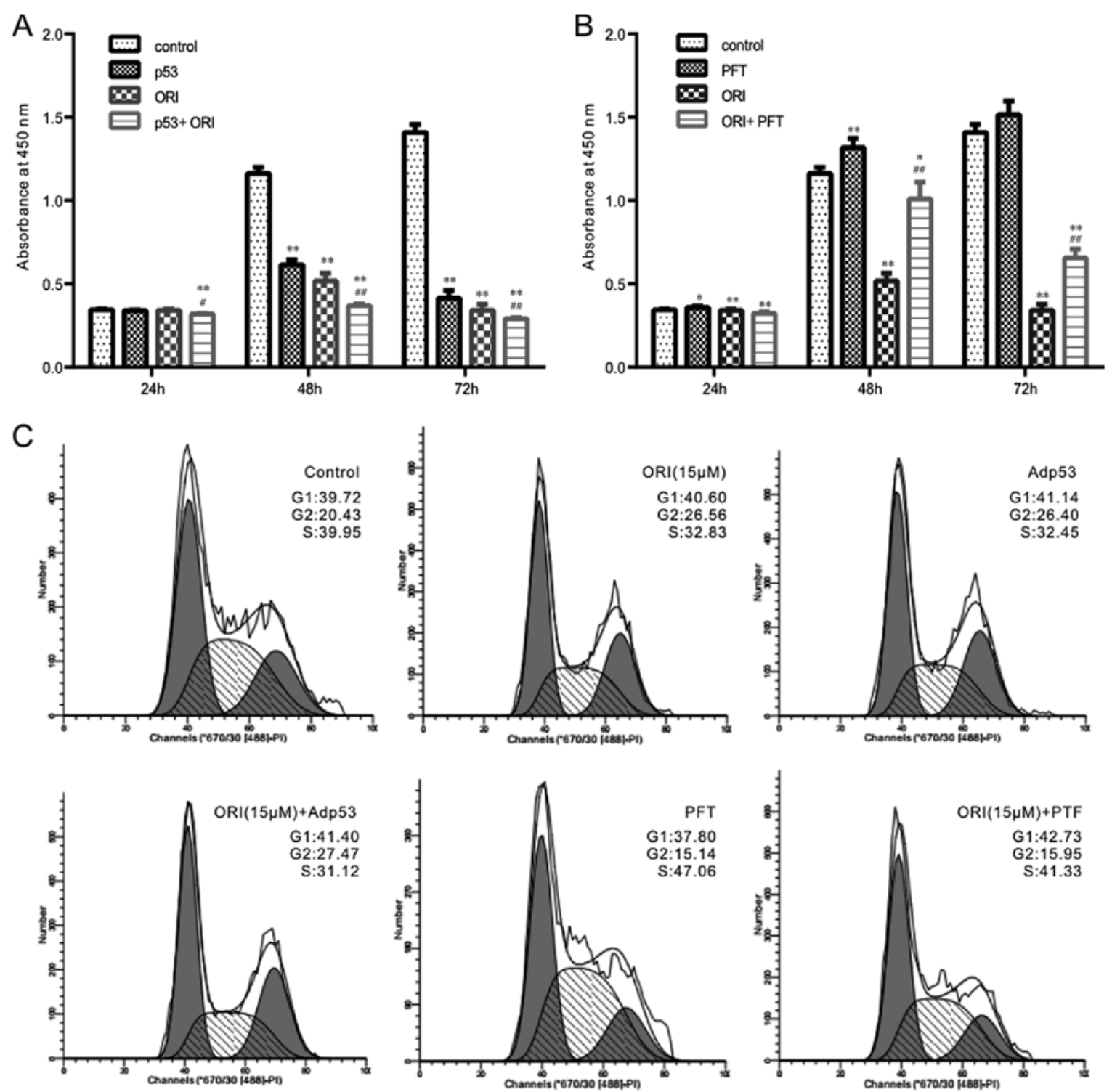

Figure 4. Effects of p53 on the anti-proliferative activity of oridonin (ORI) in SW620 cells. (A) Results of CCK-8 assay showing the effect of exogenous p53 on the anti-proliferative activity of ORI in SW620 cells $\left({ }^{* * *} \mathrm{P}<0.01\right.$ vs. control; ${ }^{~} \mathrm{P}<0.05$ vs. group treated with ORI alone; ${ }^{\# \#} \mathrm{P}<0.01$ vs. group treated with ORI alone). (B) Results of CCK-8 assay showing the effect of p53 specific inhibitor on the anti-proliferative activity of ORI in SW620 cells ("P $<0.05$ vs. control; ${ }^{* *} \mathrm{P}<0.01$ vs. control; ${ }^{\# \#} \mathrm{P}<0.01$ vs. group treated with ORI alone. PFT, p53 specific inhibitor, pifithrin- $\beta$ hydrobromide). (C) Results of flow cytometric analysis showing the effect of exogenous p53 or p53 specific inhibitor on the ORI-induced cell cycle arrest in SW620 cells.

Effects of p53 on the anti-proliferative effects of ORI on SW620 cells. The effects of p53 on the anti-proliferative activity of ORI were investigated in the colon cancer cells transfected with p53 recombinant adenoviruses or treated with a specific inhibitor (PFT, $3 \mu \mathrm{g} / \mathrm{ml}$ ). The results of the CCK-8 assay indicated that PFT markedly attenuated the p53-induced enhancement of the anti-proliferative effects of ORI against the SW620 cells (Fig. 4A and B). The results of flow cytometric analysis revealed that the combination of ORI and p53 did not markedly affect the ORI-induced cell cycle arrest; however, the p53 specific inhibitor substantially attenuated the ORI-induced G2 phase arrest in the SW620 cells (Fig. 4C). Therefore, these data indicate that the anti-proliferative activity of ORI may be partially mediated by the activation of the p53 signal.

Effects of ORI on BMP7 expression in SW620 cells. We have already established the mediatory role of p53 in the anti-proliferative effects of ORI against the SW620 cells. However, the molecular mechanisms behind these effects (ORI-induced activation of the p53 signal) remain unknown. According to our previous study, BMP7 mediated the anticancer activity of ORI against HCT116 cells (14). In this study, the potential role of ORI in upregulating BMP7 expression and the associations between BMP7 and p53 wre investigated in colon cancer cells. The results of RT-qPCR revealed that ORI upregulated the mRNA level of BMP7 in a concentrationdependent manner in the SW620 cells (Fig. 5A). The results of western blot analysis and cell immunofluorescence detection assays revealed that ORI notably increased the level of BMP7 in the SW620 cells (Fig. 5B and C). Furthermore, the results revealed that either the mRNA or protein expression of BMP7 was detectable in the colon cancer cell lines and FHC cells (normal colon epithelial cell line). Moreover, the endogenous expression of BMP7 was higher in the colon cancer cell lines 

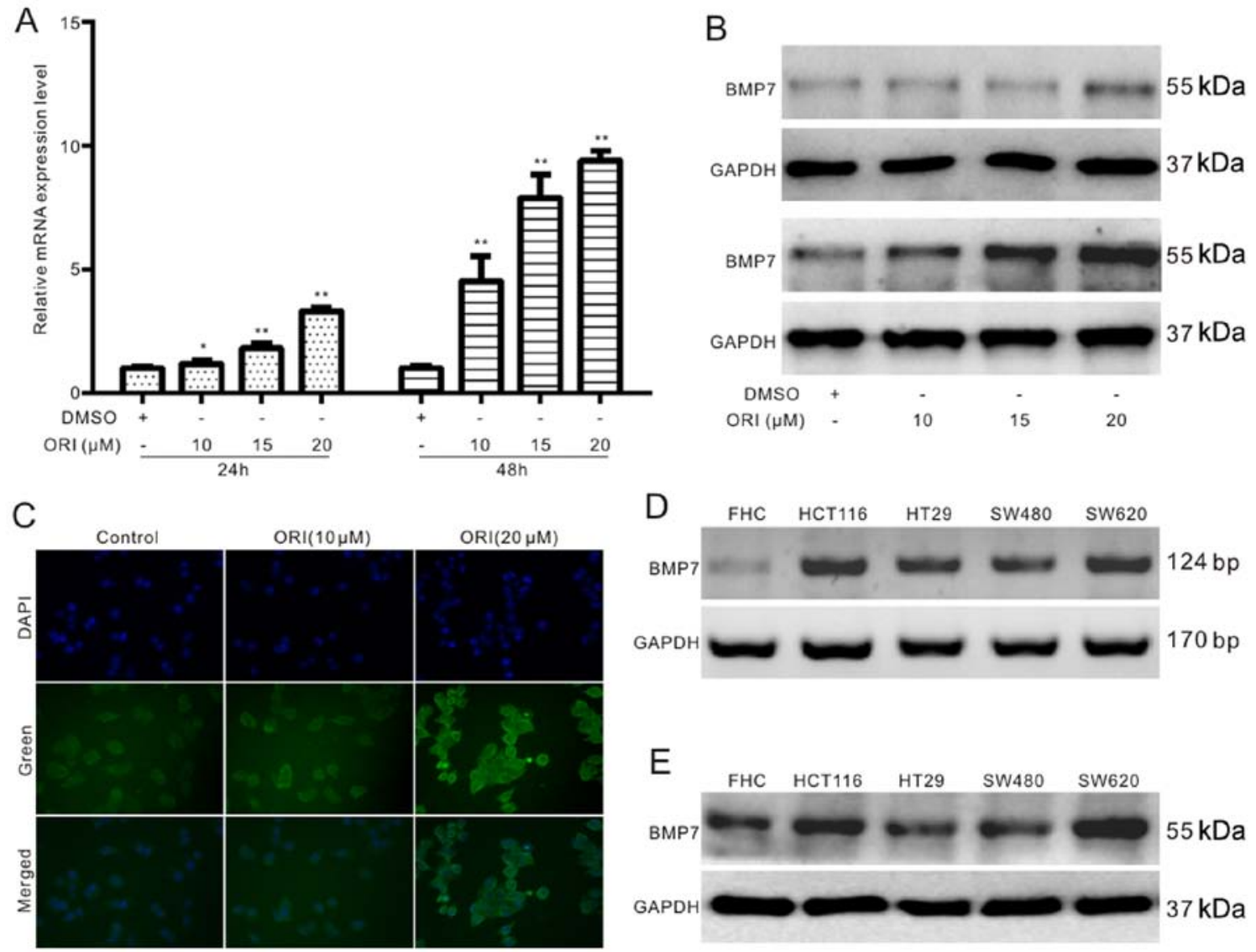

Figure 5. Effects of oridonin (ORI) on bone morphogenetic protein 7 (BMP7) in SW620 cells. (A) Results of RT-qPCR assay showing the effects of ORI on the mRNA expression of BMP7 in SW620 cells ( ${ }^{*} \mathrm{P}<0.05$ vs. control; ${ }^{* *} \mathrm{P}<0.01 \mathrm{vs}$. control). (B) Results of western blot analysis showing the effect of ORI on the protein level of BMP7 in SW620 cells. (C) Results of immunofluorescence staining showing the effect of ORI on BMP7 in SW620 cells. (D) Results of RT-qPCR showing the endogenous mRNA expression of BMP7 in colon cancer or FHC cells. (E) Results of western blot assay analysis showing the endogenous protein level of BMP7 in colon cancer or FHC cells.

than that in the FHC cells (Fig. 5D and E). All these results indicate that BMP7 may also be involved in the anticancer activity of ORI in SW620 cells.

Effects of BMP7 on the anticancer activity of ORI in SW620 cells. The effects of BMP7 on the anticancer activity of ORI were analyzed in SW620 cells. The results of the CCK-8 assay revealed that exogenous BMP7 enhanced the anti-proliferative effects of ORI; however, the BMP7-specific antibody significantly decreased the anti-proliferative effects of ORI on the SW620 cells (Fig. 6A and B). In addition, the results of our in vivo experiments revealed that the weight and size of the tumors in the mice who received a combination of ORI and BMP7 were significantly smaller than those of the mice treated with ORI alone (Fig. 6C-E). The results of H\&E-staining also revealed that BMP7 enhanced ORI-induced karyopyknosis. Thus, these data indicate that the anticancer effects of ORI may be partly mediated by the upregulation of BMP7 in SW620 cells.

Effects of ORI on p38 MAPK in SW620 cells. BMP7 exerts its physiological function through the BMP/Smad pathway (canonical BMP/Smad pathway) or non-canonical BMP/Smad pathway (24). The ORI-induced upregulation of BMP7 may thus mediate the anticancer activity of ORI in colon cancer cells. Therefore, in this study, we hypothesized that ORI may affect the canonical or non-canonical BMP/Smad signaling pathway. The results of western blot analysis did not reveal any substantial effect of ORI on the levels of Smad1/5/8 or phosphorylated Smad1/5/8 in the SW620 cells; however, ORI markedly increased the level of phosphorylated p38 in the SW620 cells without any apparent effect on the level of total p38 (Fig. 7A). We also investigated the effects of p38 on the p53 signal in SW620 cells. We employed a p38 specific inhibitor (SB203580, $7 \mu \mathrm{g} / \mathrm{ml}$ ) and found that the inhibition of p38 markedly attenuated the promoting effects of ORI on the levels of p53 and p-p53 (Fig. 7B). These data suggest that ORI exerts its anticancer effects through p38 to trigger the p53 signal in SW620 cells.

Effects of BMP7 on the activation of p38 and p53 in SW620 cells. In this study, we found that ORI can upregulate BMP7 and the p53 signal in SW620 cells and activate p38, but cannot trigger the BMP/Smad signal in SW620 cells. Thus, we hypothesized that ORI may affect the activation of the p53 signal by activating p38. The results of western blot analysis indicated that BMP7 overexpression further increased the levels of p38 and p53 induced by ORI (Fig. 8A). However, the use of a specific antibody to BMP7 almost eliminated the promoting effects of ORI on the activation of p38 and p53 (Fig. 8B). These data suggest that BMP7 mediates the anticancer effects of ORI by activating p38 and p53 successively in SW620 cells. 

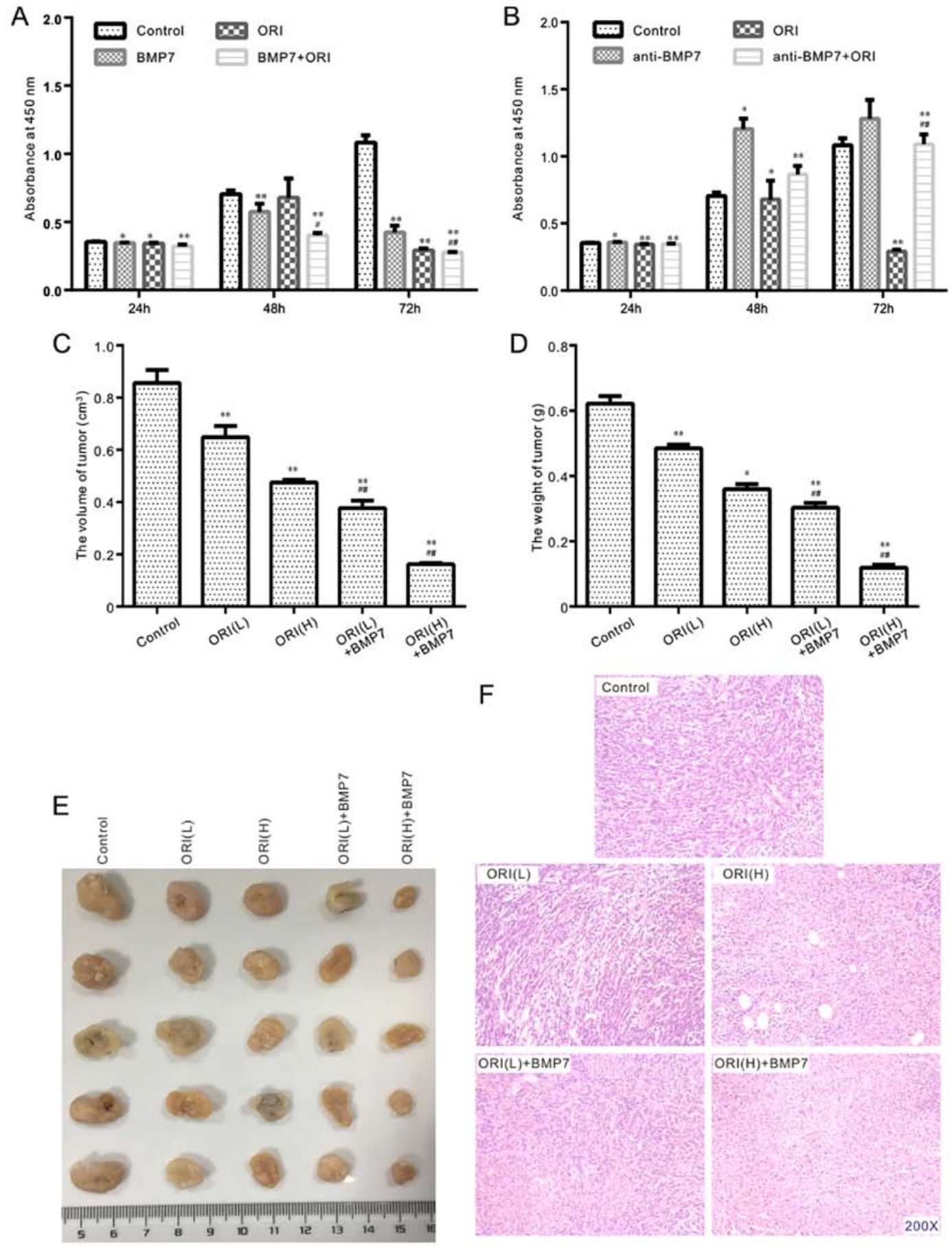

Figure 6. Effects of bone morphogenetic protein 7 (BMP7) on the anti-proliferative activity of oridonin (ORI) in SW620 cells. (A) Results of CCK-8 assay showing the effect of exogenous BMP7 on the anti-proliferative activity of ORI in SW620 cells ( ${ }^{*} \mathrm{P}<0.05$ vs. control; ${ }^{* *} \mathrm{P}<0.01$ vs. control; ${ }^{*} \mathrm{P}<0.05$ vs. groups treated with ORI alone; ${ }^{\# \#} \mathrm{P}<0.01$ vs. groups treated with ORI alone). (B) Results of CCK-8 assay showing the effect of BMP7 immunosuppression on the anti-proliferation activity of ORI in SW620 cells ("P<0.05 vs. control; ${ }^{* *} \mathrm{P}<0.01$ vs. control; ${ }^{\# \#} \mathrm{P}<0.01$ vs. groups treated with ORI alone). (C) Results of in vivo experiments showing the effect on exogenous BMP7 and/or ORI on tumor volume (D) Results of in vivo experiments showing the effect on exogenous BMP7 and/or ORI on tumor weight. (E) Representative tumor sample showing the effect of BMP7 and/or ORI on the growth of colon cancer. (F) H\&E staining results showing the anticancer effects of BMP7 and/or ORI in colon cancer.

\section{Discussion}

Colorectal cancer is a severe malignancy affecting the gastrointestinal system (25). ORI has exhibited potent anticancer properties against many forms of cancer $(5,6)$, although the specific underlying mechanisms remain unclear. In this study, the anticancer potential of ORI against colon cancer was investigated, and the findings demonstrated that BMP7 partially mediated the anticancer activity of ORI through activating the p53 signal by increasing the phosphorylation of p38 MAPK in SW620 cells.

The current therapies for colon cancer include chemotherapy, surgery and targeted therapy (26). Although the treatment regimen has been improved and optimized immensely over the past decades, the prognosis of colon cancer remains inefficient, and novel drugs with improved efficacy and safety profiles, 

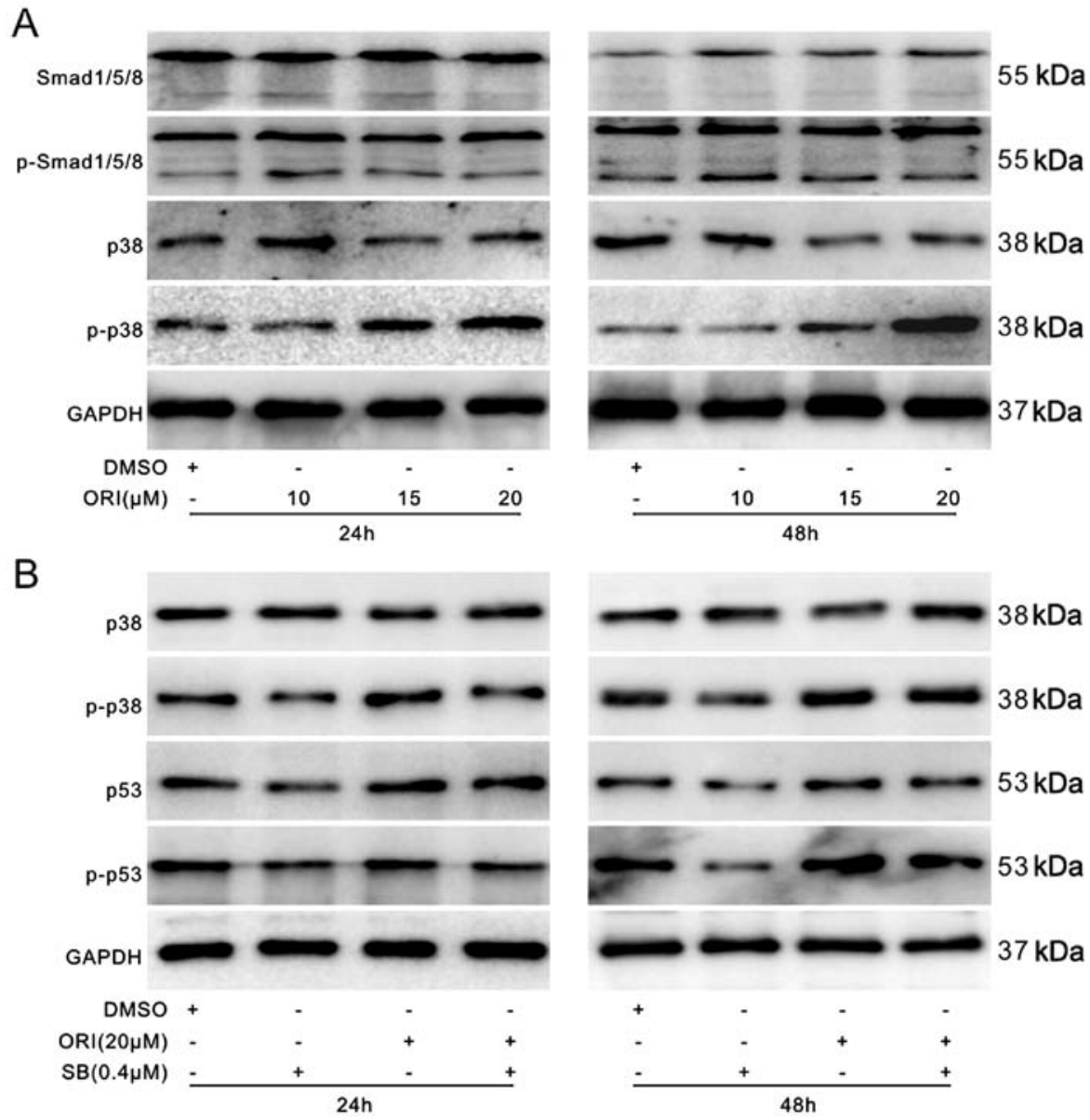

Figure 7. Effects of oridonin (ORI) on Smad1/5/8 and p38 in SW620 cells. (A) Results of western blot analysis showing the effect of ORI on the level of Smad1/5/8, p-Smad1/5/8, p38 and p-p38 in SW620 cells. (B) Results of western blot analysis showing the effect of ORI and/or p38 inhibitor on the level of p38, p-p38, p53 and p-p53 in SW620 cells (SB, p38 inhibitor SB203580).

along with novel drug targets are required for the optimal therapy of the disease. Active compounds or their derivates from traditional herbal medicine, such as camptothecin and vincristine $(3,4)$ have provided new options for cancer treatment. ORI, a diterpenoid from the traditional herbal medicine, Rasdosia rubescens, possesses anti-microbial, anti-inflammatory and antioxidant properties (27). Clinical studies have demonstrated that ORI is effective in treating esophageal, liver and breast cancer $(5,6)$. ORI has been proven to be an effective remedy for suppurative tonsillitis, acute and chronic pharyngitis, and chronic bronchitis (28). ORI also possesses long-term or moderate-acting central nervous system depressant, antihypertensive, and bidirectional regulating properties (28). ORI can be used in combination with other chemotherapeutic drugs, such as PN (pingyangmycin + detoxification) or cisplatin for enhancing the overall anticancer efficacy. ORI can inhibit the proliferation and can induce the apoptosis of different cancer cells, such as gastric, breast, pancreatic, prostate and colon cancer cells $(5,6,29,30)$. Our previous studies also demonstrated the anticancer activity of ORI against colon cancer $(7,14)$. The mechanisms underlying the effects of ORI may include the inhibition of the Wnt/ $\beta$-catenin and PI3K/Akt signal, the downregulation of Bcl-2 and epidermal growth factor receptor, and the upregulation of the p53 signal (6,31-33). However, the detailed mechanism behind the anticancer effects of ORI remain unclear.
The aberrant transduction of various signaling pathways and mutations of certain essential factors, such as TGF- $\beta$, Wnt/ $\beta$-catenin, PI3K/Akt, p53 and PTEN are associated with colon cancer (34). Different factors, such as DNA damage, oxidative stress and p38 MAPK can regulate p53 (21). The results of this study indicated that ORI substantially upregulated the p53 signal in colon cancer, and the inhibition of p53 may decrease the anti-proliferative effects of ORI. Therefore, the present study suggests that p53 partially mediates the anticancer activity of ORI; however, the exact mechanisms behind these phenomena remain unclear.

BMPs belong to TGF- $\beta$ superfamily, and play a crucial role in bone development, fracture repair, and in the pathogenesis of certain solid tumors by regulating cell differentiation, proliferation and apoptosis (9-11). Currently, 20 BMPs have been identified in human cells. The functions of BMPs in the pathogenesis of cancer may depend on the subtypes of BMPs and cancer, and even the microenvironment of the malignancy (35). BMP7 is a pleiotropic cytokine which transforms mesenchymal cells into bone and cartilage. Owing to its osteogenic activity, BMP7 has been approved by the FDA for the treatment of bone-related diseases, such as spinal fusion and bone fracture healing $(36,37)$. BMP7 has also been implicated in tumors, such as prostate and breast cancer $(38,39)$. BMP7 can reduce bone metastasis and invasive ability (40). In this study, ORI substantially upregulated BMP7 expression in 

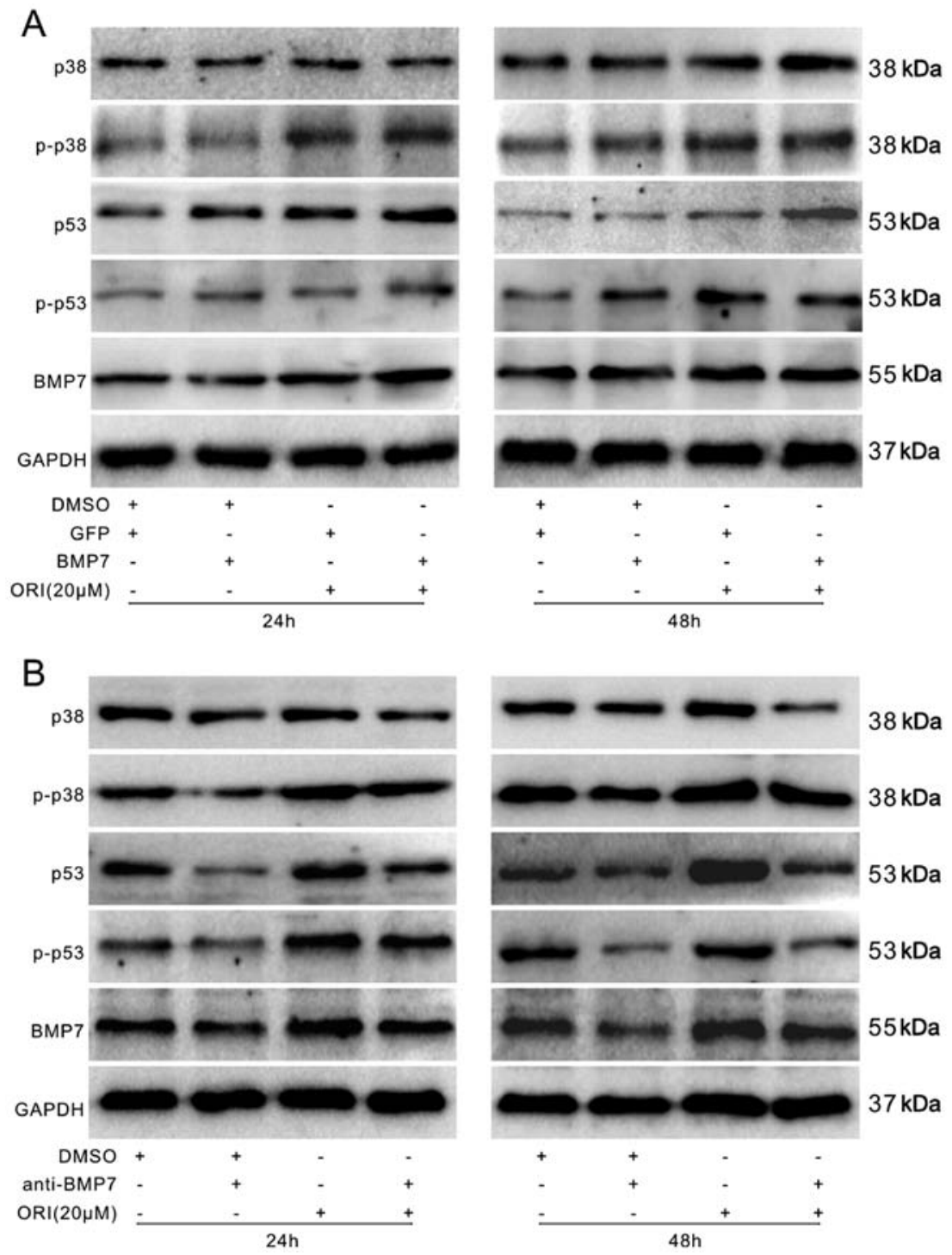

Figure 8. Effects of bone morphogenetic protein 7 (BMP7) on the oridonin (ORI)-induced p38 and p53 in SW620 cells. (A) Results of western blot analysis showing the effect of ORI and/or BMP7 on the level of p38, p-p38, p53 and p-p53 in SW620 cells. (B) Results of western blot analysis showing the effect of ORI and/or BMP7 antibody on the level of p38, p-p38, p53 and p-p53 in SW620 cells.

SW620 cells. The endogenous level of BMP7 was much higher in the colon cancer cells than in the FHC cells. The overexpression of BMP7 enhanced the anti-proliferative activity of ORI, while BMP7-specific antibody decreased the anti-proliferative effects of ORI on SW620 cells. Our findings suggest that BMP7 may be a suitable target of chemotherapeutic drugs in colon cancer. In this study, we also investigated the influence of BMP7 on $\mathrm{p} 53$.

BMP7 carries out its physiological functions through the canonical BMP/Smad pathway, or the non-canonical BMP/Smad pathway, such as MAPK and PI3K/Akt signaling. In SW620 cells, functional losses and the mutation of Smad4 are the primary causes of malignancy $(41,42)$. For this reason, non-canonical BMP/Smad signaling is responsible for the antiproliferative effects of the drug. In this study, ORI exerted no obvious effects on either the total level of Smad1/5/8 or the level of p-Smad1/5/8. ORI increased the level of p-p38 in SW620 cells, though the level of total p38 remained unaltered. These results indicate that BMP7 may mediate the anti-proliferative effects of ORI through the p38 MAPK signaling pathway.
The downregulation of p53 (a vital tumor suppressor) is a primary causative factor for colon cancer. The mutation or functional loss of p53 have been identified in $>50 \%$ of human tumor cells $(43,44)$. Stress can activate the $\mathrm{p} 35$ signal, such as p38 MAPK (21). BMP7 may activate p53 through activating p38. The data of this study suggested that the inhibition of p38 decreased the ORI-induced activation of p53 in SW620 cells. Therefore, BMP7 may mediate the anti-proliferative activity of ORI in colon cancer cells by activating the p53 signal. Further experiments indicated that the overexpression of BMP7 potentiated the ORI-induced activation of $\mathrm{p} 38$ and p53 in SW620 cells. On the contrary, the immunosuppression of BMP7 decreased the ORI-induced activation of $\mathrm{p} 38$ and p53. Hence, the upregulation of BMP7 may trigger the ORI-induced activation of p53 in colon cancer cells.

In conclusion, the results of this study suggest that ORI possesses significant anti-proliferative activity against colon cancer cell lines. The p53 signal may partially mediate the anti-proliferative effects of ORI through the BMP7/p38 pathway. 


\section{Acknowledgements}

The authors would like to thank Professor T.C. He from the University of Chicago Medical Center (Chicago, IL, USA) for providing the recombinant adenoviruses.

\section{Funding}

This study was partially supported by a research grant from the National Natural Science Foundation of China (Grants nos. NSFC 81372120 and 81572226 to Bai-Cheng He) and the grants from the Science and Technology Commission of Yuzhong, Chongqing, China (no. 20150120).

\section{Availability of data and materials}

The datasets used or analyzed during the current study are available from the corresponding author on reasonable request.

\section{Authors' contributions}

$\mathrm{BCH}$ and WJS designed the experiments; RXL, YM and XLH performed the experiments and prepared the figures; WYR analyzed the data; YPL, HW, JHZ and KW helped to perform experiments; $\mathrm{BCH}$ wrote the manuscript. All authors read and approved the final manuscript.

\section{Ethics approval and consent to participate}

For experiments involving animals, all experimental procedures were approved by the Institutional Animal Care and Use Committee of Chongqing Medical University.

\section{Consent for publication}

Not applicable.

\section{Competing interests}

The authors declare that they have no competing interests.

\section{References}

1. Applegate $\mathrm{CC}$ and Lane MA: Role of retinoids in the prevention and treatment of colorectal cancer. World J Gastrointest Oncol 7: 184-203, 2015.

2. Fellner C: Promising drugs in clinical development to treat advanced colorectal cancer. PT: 42: 262-265, 2017.

3. Banjerdpongchai R, Chanwikruy Y, Rattanapanone V and Sripanidkulchai B: Induction of apoptosis in the human Leukemic U937 cell line by Kaempferia parviflora Wall.ex.Baker extract and effects of paclitaxel and camptothecin. Asian Pac J Cancer Prev 10: 1137-1140, 2009.

4. Weaver BA: How Taxol/paclitaxel kills cancer cells. Mol Biol Cell 25: 2677-2681, 2014

5. Owona BA and Schluesener HJ: Molecular insight in the multifunctional effects of oridonin. Drugs RD 15: 233-244, 2015.

6. Liu Y, Liu YZ, Zhang RX, Wang X, Meng ZJ, Huang J, Wu K, Luo JY,Zuo GW, Chen L, et al: Oridonin inhibits the proliferation of human osteosarcoma cells by suppressing Wnt/ $\beta$-catenin signaling. Int J Oncol 45: 795-803, 2014.

7. Wu QX, Yuan SX, Ren CM, Yu Y, Sun WJ, He BC and Wu K: Oridonin upregulates PTEN through activating p38 MAPK and inhibits proliferation in human colon cancer cells. Oncol Rep 35: 3341-3348, 2016
8. Lu J, Chen X, Qu S, Yao B, Xu Y, Wu J, Jin Y and Ma C: Oridonin induces G2/M cell cycle arrest and apoptosis via the PI3K/Akt signaling pathway in hormone-independent prostate cancer cells. Oncol Lett 13: 2838-2846, 2017.

9. Bollum LK, Huse K, Oksvold MP, Bai B, Hilden VI, Forfang L, Yoon SO, Wälchli S, Smeland EB and Myklebust JH: BMP-7 induces apoptosis in human germinal center B cells and is influenced by TGF- $\beta$ receptor type I ALK5. PLoS One 12: e0177188, 2017.

10. Yoo HS, Kim GJ, Song DH, Chung KH, Lee KJ, Kim DH and An JH: Calcium supplement derived from Gallus gallus domesticus promotes BMP-2/RUNX2/SMAD5 and suppresses TRAP/RANK expression through MAPK signaling activation. Nutrients 9: E504, 2017

11. Bernatik O, Radaszkiewicz T, Behal M, Dave Z, Witte F, Mahl A, Cernohorsky NH, Krejci P, Stricker S and Bryja V: A novel role for the BMP antagonist noggin in sensitizing cells to non-canonical Wnt-5a/Ror2/disheveled pathway activation. Front Cell Dev Biol 5: 47, 2017.

12. Zhang Y, Chen X, Qiao M, Zhang BQ, Wang N, Zhang Z, Liao Z, Zeng L, Deng Y, Deng F, et al: Bone morphogenetic protein 2 inhibits the proliferation and growth of human colorectal cancer cells. Oncol Rep 32: 1013-1020, 2014.

13. Basu S, Haase G and Ben-Ze'ev A: Wnt signaling in cancer stem cells and colon cancer metastasis. F1000 Res 5: 699-709, 2016.

14. Ren CM, Li Y, Chen QZ, Zeng YH, Shao Y, Wu QX, Yuan SX, Yang JQ, Yu Y, Wu K, et al: Oridonin inhibits the proliferation of human colon cancer cells by upregulating BMP7 to activate p38 MAPK. Oncol Rep 35: 2691-2698, 2016.

15. Briest F and Grabowski P: The p53 network as therapeutic target in gastroenteropancreatic neuroendocrine neoplasms. Cancer Treat Rev 41: 423-430, 2015.

16. Lu L, Harutyunyan K, Jin W, Wu J, Yang T, Chen Y, Joeng KS, Bae Y, Tao J, et al: RECQL4 regulates p53 function in vivo during skeletogenesis. J Bone Miner Res 30: 1077-1089, 2015.

17. Rajeshkumar NV, Dutta P, Yabuuchi S, de Wilde RF, Martinez GV, Le A, Kamphorst JJ, Rabinowitz JD, Jain SK, Hidalgo M, et al: Therapeutic targeting of the warburg effect in pancreatic cancer relies on an absence of p53 function. Cancer Res 75: 3355-3364, 2015.

18. Inobe $\mathrm{T}$, Nozaki $\mathrm{M}$ and Nukina $\mathrm{N}$ : Artificial regulation of $\mathrm{p} 53$ function by modulating its assembly. Biochem Biophys Res Commun 467: 322-327, 2015.

19. Gan F, Zhou Y, Hou L, Qian G, Chen X and Huang K: Ochratoxin A induces nephrotoxicity and immunotoxicity through different MAPK signaling pathways in PK15 cells and porcine primary splenocytes. Chemosphere 182: 630-637, 2017.

20. Koul HK, Pal M and Koul S: Role of p38 MAP Kinase Signal Transduction in Solid Tumors. Genes Cancer 4: 342-359, 2013.

21. Kim GT, Lee SH, Kim JI and Kim YM: Quercetin regulates the sestrin 2-AMPK-p38 MAPK signaling pathway and induces apoptosis by increasing the generation of intracellular ROS in a p53-independent manner. Int J Mol Med 33: 863-869, 2014.

22. Luo J, Deng ZL, Luo X, Tang N, Song WX, Chen J, Sharff KA, Luu HH, Haydon RC, Kinzler KW, et al: A protocol for rapid generation of recombinant adenoviruses using the AdEasy system. Nat Protoc 2: 1236-1247, 2007.

23. Livak KJ and Schmittgen TD: Analysis of relative gene expression data using real-time quantitative PCR and the 2(-Delta Delta C(T)) method. Methods 25: 402-408, 2001.

24. Stayce EB, Barbara HJ, Antonio F, Jessica G, Eunice DR, Betty LC, Sherry CH, Jimmy YC and John MC: Bone morphogenetic protein signaling and growth suppression in colon cancer. Am J Physiol Gastrointest Liver Physiol 291: G135-G145, 2006.

25. Carvalho $\mathrm{C}$ and Glynne-Jones R: Challenges behind proving efficacy of adjuvant chemotherapy after preoperative chemoradiation for rectal cancer. Lancet Oncol 18: e354-e363, 2017.

26. Link KH, Coy P, Roitman M, Link C, Kornmann M and Staib L: Minimum volume discussion in the treatment of colon and rectal cancer: A review of the current status and relevance of surgeon and hospital volume regarding result quality and the impact on health economics. Visc Med 33: 140-147, 2017.

27. Li D, Han T, Liao J, Hu X, Xu S, Tian K, Gu X, Cheng K, Li Z, Hua $\mathrm{H}$, et al: Oridonin, a promising ent-kaurane diterpenoid lead compound. Int J Mol Sci 17: E1395, 2016.

28. Xu J, Wold EA, Ding Y, Shen Q and Zhou J: Therapeutic potential of oridonin and its analogs: From anticancer and antiinflammation to neuroprotection. Molecules 23: E474, 2018.

29. Wu J, Ding Y, Chen CH, Zhou Z, Ding C, Chen H, Zhou J and Chen C: A new oridonin analog suppresses triple-negative breast cancer cells and tumor growth via the induction of death receptor 5. Cancer Lett 380: 393-402, 2016. 
30. Gui Z, Luo F, Yang Y, Shen C, Li S and Xu J: Oridonin inhibition and $\mathrm{miR} 200 \mathrm{~b} 3 \mathrm{p} / \mathrm{ZEB} 1$ axis in human pancreatic cancer. Int $\mathrm{J}$ Oncol 50: 111-120, 2017.

31. Zhang LD, Liu Z, Liu H, Ran DM, Guo JH, Jiang B, Wu YL and Gao FH: Oridonin enhances the anticancer activity of NVP-BEZ235 against neuroblastoma cells in vitro and in vivo through autophagy. Int J Oncol 49: 657-665, 2016.

32. Pi J, Jin H, Jiang J, Yang F, Cai H, Yang P, Cai J and Chen ZW: Single molecule force spectroscopy for in-situ probing oridonin inhibited ROS-mediated EGF-EGFR interactions in living KYSE-150 cells. Pharmacol Res 119: 479-489, 2017.

33. Yao Z, Xie F, Li M, Liang Z, Xu W, Yang J, Liu C, Li H, Zhou H and $\mathrm{Qu} \mathrm{LH}$ : Oridonin induces autophagy via inhibition of glucose metabolism in p53-mutated colorectal cancer cells. Cell Death Dis 8: e2633, 2017

34. Arvelo F, Sojo F and Cotte C: Biology of colorectal cancer. Ecancermedicalscience 9: 520, 2015.

35. Lochab AK and Extavour CG: Bone morphogenetic protein (BMP) signaling in animal reproductive system development and function. Dev Biol 427: 258-269, 2017.

36. Tsuji K, Cox K, Gamer L, Graf D, Economides A and Rosen V: Conditional deletion of BMP7 from the limb skeleton does not affect bone formation or fracture repair. J Orthop Res 28: 384-389, 2010.

37. Wang Z, Fu C, Chen Y, Xu F, Wang Z, Qu Z and Liu Y: FoxC2 enhances BMP7-mediated anabolism in nucleus pulposus cells of the intervertebral disc. PLoS One 11: e0147764, 2016.
38. Lim M, Chuong CM and Roy-Burman P: PI3K, Erk signaling in BMP7-induced epithelial-mesenchymal transition (EMT) of PC-3 prostate cancer cells in 2- and 3-dimensional cultures. Horm Cancer 2: 298-309, 2011

39. Rodriguez-Martinez A, Alarmo EL, Saarinen L, Ketolainen J, Nousiainen K, Hautaniemi S and Kallioniemi A: Analysis of BMP4 and BMP7 signaling in breast cancer cells unveils time-dependent transcription patterns and highlights a common synexpression group of genes. BMC Med Genomics 4: 80, 2011.

40. Buijs JT, Henriquez NV, van Overveld PG, van der Horst G, ten Dijke P and van der Pluijm G: TGF-beta and BMP7 interactions in tumour progression and bone metastasis. Clin Exp Metastasis 24: 609-617, 2007

41. Yan P, Klingbiel D, Saridaki Z, Ceppa P, Curto M, McKee TA, Roth A, Tejpar S, Delorenzi M, Bosman FT, et al: Reduced expression of SMAD4 is associated with poor survival in colon cancer. Clin Cancer Res 22: 3037-3047, 2006.

42. Zhang B, Halder SK, Kashikar ND, Cho YJ, Datta A, Gorden DL and Datta PK: Antimetastatic role of Smad4 signaling in colorectal cancer. Gastroenterology 138: 969-980.e961-963, 2010.

43. Vousden KH and Lane DP: p53 in health and disease. Nat Rev Mol Cell Biol 8: 275-283, 2007.

44. Nayak D, Kumar A, Chakraborty S, Rasool RU, Amin H, Katoch A, Gopinath V, Mahajan V, Zilla MK, Rah B, et al: Inhibition of Twist1-mediated invasion by Chk 2 promotes premature senescence in p53-defective cancer cells. Cell Death Differ 24: 1275-1287, 2017. 